Artículo

\title{
Variabilidad genética en raíz de girasol mediante gamma de ${ }^{60} \mathrm{Co}$
}

\author{
Humberto Rafael Bravo Delgado ${ }^{1}$ \\ Ernesto Díaz-López ${ }^{2 \S}$
}

${ }^{1}$ Cuerpo Académico Procesos Bioalimentarios-Universidad Tecnológica de Tehuacán. Prolongación del 1 sur 1101, San Pablo Tepetzingo, Tehuacán, Puebla. CP. 75859. (rafael.bravo@ uttehuacan.edu.mx). ${ }^{2}$ Cuerpo Académico Ecofisiología Aplicada a Cultivos en Zonas Áridas-Universidad Tecnológica de Tehuacán. Prolongación del 1 sur 1101, San Pablo Tepetzingo, Tehuacán, Puebla. CP. 75859.

${ }^{\S}$ Autor para correspondencia: ernesto.lopez@uttehuacan.edu.mx.

\section{Resumen}

El girasol es un cultivo que pertenece a la familia asteraceae. Esta planta posee múltiples usos dentro de los cuales se pueden citar: extracción de aceite de sus semillas, ornamental y debido al gran volumen del sistema radical como planta biorremediadora. Lo anterior, es ocasionado por la capacidad de extracción de metales pesados tales como: $\mathrm{Pb}, \mathrm{Mg}$ y $\mathrm{Ca}$, que afectan los suelos agrícolas. El objetivo de la presente investigación fue: inducir variabilidad genética en el sistema radical del girasol, mediante el uso de irradiación gamma de ${ }^{60} \mathrm{Co}$. Para ello se sembraron aquenios en condiciones de invernadero en macetas de $4 \mathrm{~kg}$ de capacidad durante el verano de 2019 en el Instituto Nacional de Investigaciones Nucleares ubicado en la Marquesa, México, los cuales fueron evaluados bajo un diseño completamente al azar. Los tratamientos consistieron en once niveles de irradiación gamma de ${ }^{60} \mathrm{Co}$ y cuatro repeticiones $(11$ x 5$)=55$ unidades experimentales. Los resultados indican que el mayor volumen, longitud y biomasa seca de raíz, se obtuvieron con la aplicación de 200 Gy de irradiación gamma de ${ }^{60} \mathrm{Co}$. La LD $\mathrm{LD}_{50}$ para germinación se alcanzó a 207 Gy, mientras que para brotación con 183 Gy. De este trabajo se concluye que la aplicación de 200 Gy de ${ }^{60} \mathrm{Co}$, pueden inducir variabilidad genética en atributos deseables en el girasol, cuando este es destinado a la biorremediación de suelos agrícolas.

Palabras clave: biorremediación, plasticidad fenotípica, Valle de Tehuacán, volumen radical.

Recibido: febrero de 2021

Aceptado: abril de 2021 


\section{Introducción}

El girasol (Helianthus annuus L.) es un cultivo que por muchos años se ha considerado como oleaginoso, debido a que de sus semillas se extraen ácidos grasos de alta calidad, para el consumo humano. Sin embargo, en la actualidad, se está utilizando como una planta biorremediadora de suelos Escalante et al. (2017), a causa del gran desarrollo de su sistema radical, así como a la capacidad de este para absorber cationes del suelo tales como: $\mathrm{Ca}^{++}, \mathrm{Mg}^{++}$y Na $\mathrm{Na}^{+}$, incluso metales pesados como el plomo y cadmio (Chico et al., 2012; Suaña, 2018).

Este cultivo, presenta una amplia plasticidad fenotípica para adaptarse a suelos agrícolas, donde existen problemas de alcalinidad, debido a la irrigación de estos con aguas duras, como en el valle de Tehuacán, donde estas prácticas han causado el deterioro de algunos parámetros químicos y físicos del suelo, tales como: $\mathrm{pH}$ alcalino, altas conductividades eléctricas y suelos defloculados. Cardoso et al. (2018), encontraron que las plantas de girasol cultivadas en condiciones limitadas de agua ajustaban significativamente el potencial osmótico en la hoja, lo que se relacionaba con una prolongación de la abertura estomatal a medida que el suelo se secaba y una menor sensibilidad de la fotosíntesis a los daños inducidos por el estrés hídrico.

En estudios recientes enfocados a cuestiones morfológicas, se han demostrado, que el sistema radical de esta asterácea puede llegar a alcanzar hasta $1.5 \mathrm{~m}$ de longitud y presentar un alto volumen radical, lo que permite gran absorción de agua y nutrimentos, siendo considerada como una planta por excelencia, para estudios de fisiología de la raíz.

A este respecto Díaz et al. (2017), estudiaron la dinámica del pH y conductividad eléctrica, de un suelo irrigado con aguas duras cuando es sembrado con girasol y mencionan que éste, puede soportar hasta un $\mathrm{pH}$ de 10.5 y $6.5 \mathrm{dS} \mathrm{m}^{-1}$ de conductividad, además de absorber $160 \mathrm{mg} \mathrm{L}^{-1}$ de calcio, considerándolo como una planta con potencial, de bajar el $\mathrm{pH}$. Por su parte Escalante et al. (2017), mencionan que las plántulas de girasol, tiene la capacidad de soportar altos potenciales

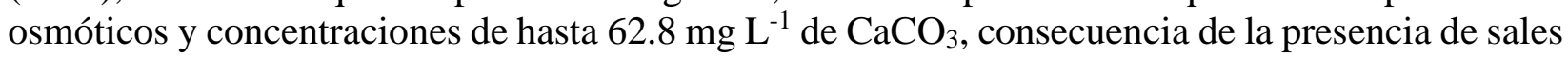
de $\mathrm{Ca}++$ en la solución del suelo, que otras especies no soportarían.

Por ello el objetivo general de la presente investigación fue: inducir variabilidad genética en los parámetros longitud y volumen del sistema radical de girasol, mediante el uso de radiación gamma de ${ }^{60} \mathrm{Co}$. La hipótesis fue: la irradiación gamma de ${ }^{60} \mathrm{Co}$, inducirá variabilidad genética en dosis bajas, cuando los aquenios son sometidos a esta.

\section{Materiales y métodos}

La presente investigación, se llevó a cabo en el Instituto Nacional de Investigaciones Nucleares (ININ), ubicado en Ocoyoacac, México, en un invernadero tipo Mini-Green. El germoplasma consistió de aquenios de girasol, $c v$ Periquero (Morales et al., 2015), que corresponde a un material de polinización libre, con tres ciclos de selección masal.

Los tratamientos consistieron en 11 dosis de radiación gamma de ${ }^{60} \mathrm{Co}, 0 ; 100 ; 200 ; 300 ; 400 ; 500$; 600; 700; 800; 900 y 1000 Gy, que fueron irradiados en el transelektro modelo LGI-01, el 6 de junio de 2019 en una razón de dosis actual de $697.85 \mathrm{~Gy} \mathrm{~h}^{-1}$, de acuerdo con el departamento del 
irradiador del ININ. La unidad experimental se constituyó, por una maceta de $4 \mathrm{~kg}$ de capacidad y seis plántulas de girasol, siendo tres plántulas la parcela útil. El sustrato utilizado consistió de suelo del sitio y materia orgánica a una proporción (50:50) v/v, las propiedades físicas y químicas del mismo, se presentan en la Cuadro 1.

Cuadro 1. Propiedades físicas y químicas del sustrato de evaluación de girasol (Helianthus annuus L.) $c v$ Periquero. Instituto Nacional de Investigaciones Nucleares, 2019.

\begin{tabular}{cccc}
\hline Propiedades físicas & \multicolumn{3}{c}{ Propiedades químicas } \\
\hline Densidad aparente & $1.15 \mathrm{~g} \mathrm{~cm}^{-3}$ & $\mathrm{pH}$ & 5.5 \\
Textura & Franco arcillo limoso & $\mathrm{CE}$ & $1.3 \mathrm{dS} \mathrm{m}^{-1}$ \\
Velocidad de percolación & $1.1 \mathrm{~cm} \mathrm{~s}^{-1}$ & $\mathrm{MO}$ & $3.5 \%$ \\
Color & Seco 5YR 4/2 & Nitrógeno & $13.5 \mathrm{mg} \mathrm{kg}^{-1}$ \\
& Mojado 5YR 3/2 &
\end{tabular}

$\mathrm{pH}=$ potencial de hidrógeno; $\mathrm{CE}=$ conductividad eléctrica; $\mathrm{MO}=$ materia orgánica.

El diseño experimental fue completamente aleatorizado (DCA) y cinco repeticiones $(11 \times 5)=55$ unidades experimentales, que fueron evaluadas bajo el modelo matemático Yij $=\mu+\mathrm{Ti}+$ cij. Donde: Yij, es la variable respuesta de la i-ésima dosis de iradiación en la j-ésima repetición; $\mu$, es la media general verdadera; Ti, es el efecto de la i-ésima dosis de iradiación gamma de ${ }^{60} \mathrm{Co}$ y $\varepsilon \mathrm{ij}$, es el error experimental de la i-ésima dosis de iradiación en la j-ésima repetición (Steel y Torrie, 1996; Infante y Zárate, 2012).

Las variables respuesta fueron: porcentaje de germinación, para ello se sembraron diez aquenios en cajas Petri de plástico de $10 \mathrm{~cm}$ de diámetro, previamente desinfectados en una solución de hipoclorito de calcio al 1\%, durante 5 min.

Para la retención de humedad en el fondo de la caja Petri, se colocó un fondo de papel filtro con agua destilada. Una vez constituida la unidad experimental, estas fueron colocadas en una estufa a $20{ }^{\circ} \mathrm{C}$, hasta que la radícula rompió el pericarpio, (Taiz y Zeiger, 2000).

El porcentaje de germinación se determinó por medio de la ecuación $\mathrm{PG}=[\mathrm{ss} / \mathrm{sg}] \mathrm{x} 100$. Donde: $\mathrm{PG}=$ es el porcentaje de germinación; $\mathrm{SS}=$ semillas sembradas y $\mathrm{SG}=$ semillas germinadas (Loeza et al., 2013). Porcentaje de brotación, determinado por la relación $\mathrm{PB}=[\mathrm{PB} / \mathrm{SS}] \times 100$. Donde: $\mathrm{PB}=$ porcentaje de brotación; $\mathrm{PB}=$ plantas brotadas y $\mathrm{SS}=$ semillas sembradas. Volumen de raíz, este se calculó por medio del principio de Arquimedes, colocando el sistema radical de laplántula, en una probeta de $20 \mathrm{ml}$ con un volumen de agua destilada conocido $(10 \mathrm{ml})$, siendo el volumen radical el volumen de agua desplazado en $\mathrm{cm}^{3}$.

Longitud de raíz, midiendo esta desde la cofia de la raíz axonomorfa, hasta el inicio del hipocótilo con un vernier de acero inoxidable con una precisión de $+/-0.03 \mathrm{~mm}$, para expresar el resultado en $\mathrm{cm}$. Para las dos variables anteriores, fue necesario realizar muestreos destructivos.

Las variables fenológicas días a brotación y germinación, se determinaron contabilizando losdías a los cuales ocurrieron dichos estados fenológicos. Para este estudio, fue necesario realizar la curva de radiosensibilidad y por medio de una interpolación, determinar la dosis letal media de radiación 
(LD50) para germinación y brotación, para ello se realizó un modelo de regresión cuadrático mediante mínimos cuadrados. El porcentaje de sobrevivencia se calculó con la ecuación $\mathrm{PS}=[\mathrm{PB}$, PV] x 100. Donde: $\mathrm{PS}=$ porcentaje de sobrevivencia; $\mathrm{PB}=$ plantas brotadas y $\mathrm{PV}=$ plántulas vivas, para esta variable de igual modo se determinó la $\mathrm{LD}_{50}$.

Otra variable evaluada fue, biomasa seca total, determinada para raíz, tallo y nomófilos, sometiendo estos a secado en una estufa modelo $6 \mathrm{M}$, durante $72 \mathrm{~h}$, hasta alcanzar el peso constante (Escalante, 2017), unidades SPAD, medidas con un medidor de unidades SPAD Minolta-502, tomando lecturas directamente de la lámina foliar de cinco nomófilos a los 45 dds. Cuando las variables respuesta resultaron significativas, se les aplicó la prueba de comparación múltiple de Tukey, a un nivel de significancia de 5\% de probabilidad de error.

\section{Resultados y discusión}

\section{Curva de radiosensibilidad para germinación, brotación y LD50}

En la Figura 1, se muestra la dinámica de la curva de radiosensibilidad de aquenios de girasol cv. Periquero, sometidos a irradiación gamma de ${ }^{60} \mathrm{Co}$. En ella se puede apreciar que la germinación tuvo una tendencia a disminuir, a medida que la dosis de irradiación aumentó, ajustándose así a un modelo cuadrático, con un coeficiente de determinación altamente significativo, el cual indica, que 96\% de la germinación se debe al incremento en la irradiación gamma. De esta curva, el decaimiento drástico inició a partir de 700 Gy con un porcentaje de germinación de 4\%. La interpolación cuadrática del modelo muestra que la LD50 ocurre con la aplicación de 207 Gy.

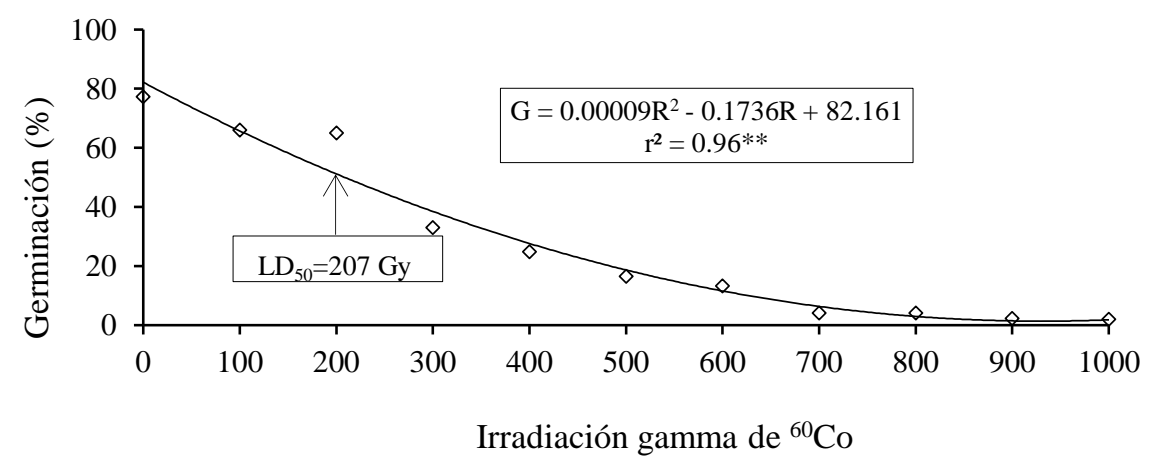

Figura 1. Dinámica de la germinación, en aquenios de girasol (Helianthus annuus L.), sometidos a 11 niveles de irradiación gamma de ${ }^{60} \mathrm{Co}$. Instituto Nacional de Investigaciones Nucleares. Ocoyoacác, México, 2019. B= brotación; y R= radiación; ${ }^{* *}$; *; n.s= significativo al 0.01; 0.05 y no significativo.

Estos resultados difieren a los reportados por Díaz et al. (2017), quienes mencionan que el modelo de ajuste cuando los aquenios de girasol $c v$ Victoria, son sometidos a irradiación gamma de ${ }^{60} \mathrm{Co}$, es polinómico de grado tres, con un coeficiente de determinación de 0.99 altamente significativo.

Estas diferencias, se atribuyen a los diferentes cultivares utilizados, así como a las condiciones climáticas contrastantes de ambos sitios de estudio. Además, el modelo matemático propuesto para irradiación vs germinación solo fue utilizado para describir la dinámica de germinación y no para, la determinación de la LD50. 
Con relación a la dosis letal media obtenida en este estudio, se encontró diferencia con lo reportado por Álvarez et al. (2018), quienes mencionan una LD50 para Eragrostis lehmanniana es de 2076 Gy, diferencias muy discrepantes, que se atribuyen a la diferencia de especies y al contenido de agua, entre otras variables

La brotación tuvo una tendencia similar a la germinación, ajustándose a un modelo cuadrático, para el mismo intervalo de irradiación (0-1 000 Gy), disminuyendo la brotación drásticamente a 700 Gy, así la interpolación para determinar la LD50 en brotación, se alcanzó a 183 Gy, 11.59\% menos que la germinación,lo que indica que la brotación en girasol $c v$ Periquero resulta ser más sensible a la irradiación gamma de ${ }^{60}$ Co que la germinación (Figura 2).

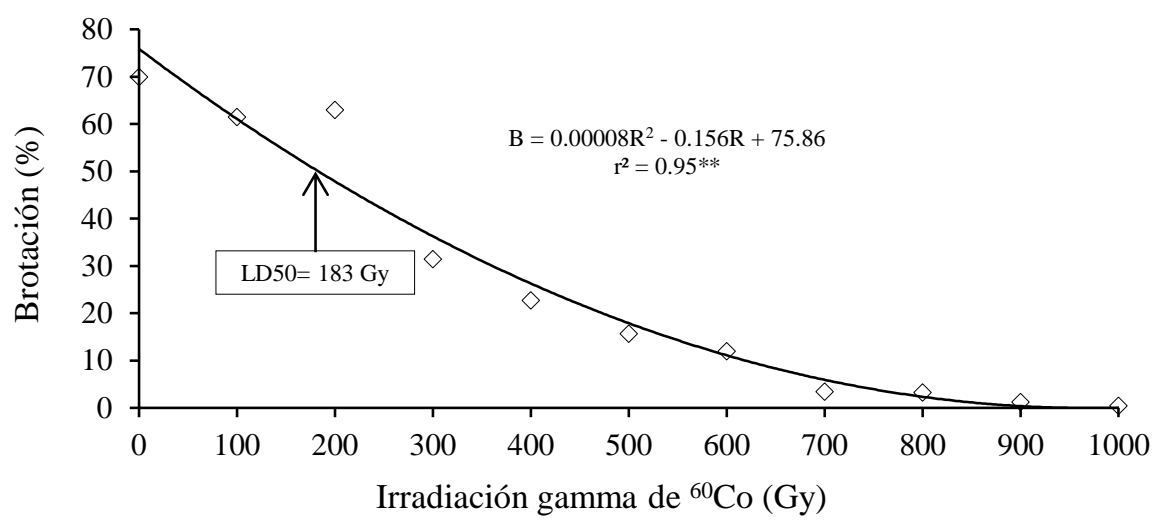

Figura 2. Dinámica de la brotación, en aquenios de girasol sometidos a 11 niveles de irradiación gamma de ${ }^{60} \mathrm{Co}$. Instituto Nacional de Investigaciones Nucleares. Ocoyoacác, México, 2019. $B=$ brotación; $R=$ radiación; ; ; ; ns= significativo al $0.01 ; 0.05$ y no significativo.

\section{Índice de corrección entre germinación vs brotación}

Al realizar el modelo de regresión lineal con ordenada al origen, entre la germinación vs la brotación, éste tuvo un ajuste lineal $\mathrm{B}=0.9307 \mathrm{G}$, con un coeficiente de determinación altamente significativo, así la pendiente del modelo, 0.9307 puede ser utilizada como coeficiente para la estimación de la germinación, mediante la brotación (De la Cruz et al., 2019) (Figura 3).

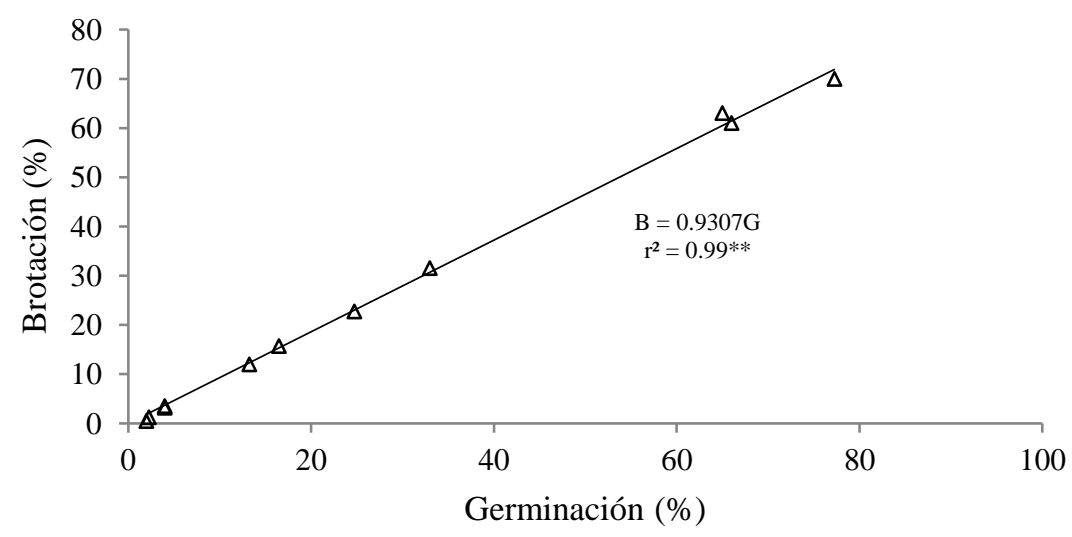

Figura 3. Coeficiente de corrección para germinación vs brotación, de aquenios de (Helianthus anuuus L.), $c v$ Periqueros sometidos a once niveles de radiación gamma de ${ }^{60} \mathrm{Co}$. Instituto Nacional de Investigaciones Nucleares, 2019. G= germinación; y B= brotación. 


\section{Porcentaje de germinación, brotación y días a germinación y brotación}

En el Cuadro 2 se presenta el análisis de varianza y prueba de comparación múltiple para porcentaje de germinación y brotación, así como para días a germinación y brotación. El análisis de varianza indicó, que existieron diferencias altamente significativas para las variables en estudio, así el coeficiente de determinación para todas las variables osciló entre 3.35 y 5.4 para días a brotación, germinación y porcentaje de brotación, mostrando así que los datos fueron muy confiables, mientras que para porcentaje de germinación el coeficiente de variabilidad $27.65 \%$, muestra que los datos son confiables.

Cuadro 2. Análisis de varianza y prueba de comparación múltiple, para cuatro parámetros en aquenios de girasol (Helianthus annuus L.), sometidos a once niveles de irradiación gamma de ${ }^{60}$ Co. Instituto Nacional de Investigaciones Nucleares, 2019.

\begin{tabular}{|c|c|c|c|c|}
\hline Irradiación & PG & PB & DG & DB \\
\hline Gy & \multicolumn{2}{|c|}{$(\%)$} & \multicolumn{2}{|c|}{ Días } \\
\hline 0 & $77.25 \mathrm{a}$ & $70 \mathrm{a}$ & $7.75 \mathrm{c}$ & $11.75 \mathrm{c}^{\mathrm{dI}}$ \\
\hline 100 & $66 a$ & $61.5 \mathrm{~b}$ & $7.25 \mathrm{~d}$ & $11.5 \mathrm{c}$ \\
\hline 200 & $65 \mathrm{a}$ & $63 \mathrm{~b}$ & $6.25 \mathrm{e}$ & $9.75 \mathrm{~d}$ \\
\hline 300 & $33 \mathrm{~b}$ & $31.5 \mathrm{c}$ & $8 \mathrm{c}$ & $11.75 \mathrm{c}$ \\
\hline 400 & $24.75 b$ & $22.75 \mathrm{~d}$ & $9 \mathrm{ab}$ & $12 \mathrm{bc}$ \\
\hline 500 & $16.5 \mathrm{~b}$ & $15.75 \mathrm{e}$ & $9.5 \mathrm{ab}$ & $12.25 \mathrm{~b}$ \\
\hline 600 & $13.25 \mathrm{bc}$ & $12 \mathrm{f}$ & $10 \mathrm{a}$ & $12.75 \mathrm{ab}$ \\
\hline 700 & $4 \mathrm{bc}$ & $3.5 \mathrm{~g}$ & $9.75 \mathrm{a}$ & $12.75 \mathrm{ab}$ \\
\hline 800 & $4 \mathrm{bc}$ & $3.25 \mathrm{~g}$ & $10 \mathrm{a}$ & $13 \mathrm{a}$ \\
\hline 900 & $2.25 \mathrm{bc}$ & $1.25 \mathrm{gh}$ & $10 \mathrm{a}$ & $13 \mathrm{a}$ \\
\hline 1000 & $2 \mathrm{bc}$ & $0.5 \mathrm{gh}$ & $10 \mathrm{a}$ & $13 \mathrm{a}$ \\
\hline DSH & $19.25^{* *}$ & $2.02^{* *}$ & $0.5^{* *}$ & $0.588^{* *}$ \\
\hline $\mathrm{CV} \%$ & 27.65 & 5.4 & 3.92 & 3.35 \\
\hline
\end{tabular}

$\mathbb{I}=$ medias dentro de columna con la misma literal, estadísticamente son iguales según Tukey a $p \leq 0.05$; PG, porcentaje de germinación; $\mathrm{PB}=$ porcentaje de brotación; $\mathrm{DG}=$ días a germinación; $\mathrm{DB}=$ días a brotación; $\mathrm{DSH}=$ diferencia significativa honesta; $\mathrm{CV}=$ coeficiente de variabilidad; ${ }^{* *} ;{ }^{*} ; \mathrm{ns}=$ significativo al $0.01 ; 0.05$ y no significativo.

Respecto a la prueba de comparación múltiple, ésta indica que el mayor porcentaje de germinación ocurrió con la aplicación de 100 y 200 Gy, incluido el testigo con 66, 65 y 77.25\%. La mayor brotación, fue en el testigo con 70\%. En relación con los días a germinación y brotación, los mayores valores fueron para las dosis altas, 800, 900 y 1000 Gy con 10 y 13 días respectivamente, esto muestra que la aplicación de altas dosis de irradiación gamma de ${ }^{60} \mathrm{Co}$, retrasan tanto la germinación como la brotación, como lo han demostrado Antúnez et al. (2017), quienes al aplicar 300 Gy de irradiación gamma de ${ }^{60} \mathrm{Co}$, alcanzaron una emergencia de plántulas a los 11 días después de la siembra, en Physalis peruviana L. 


\section{Porcentaje de sobrevivencia}

El porcentaje de sobrevivencia fue un reflejo del porcentaje de germinación y brotación, ya que este de igual modo, tuvo una tendencia a disminuir a medida que la irradiación aumentó. El modelo de ajuste fue cuadrático (Figura 4), presentando una caída drástica a 700 Gy. Para este caso, la LD50, fue a 374 Gy donde el mayor porcentaje de sobrevivencia ocurrió en el tratamiento testigo con $90 \%$ siendo este la ordenada al origen, como lo muestra el modelo.

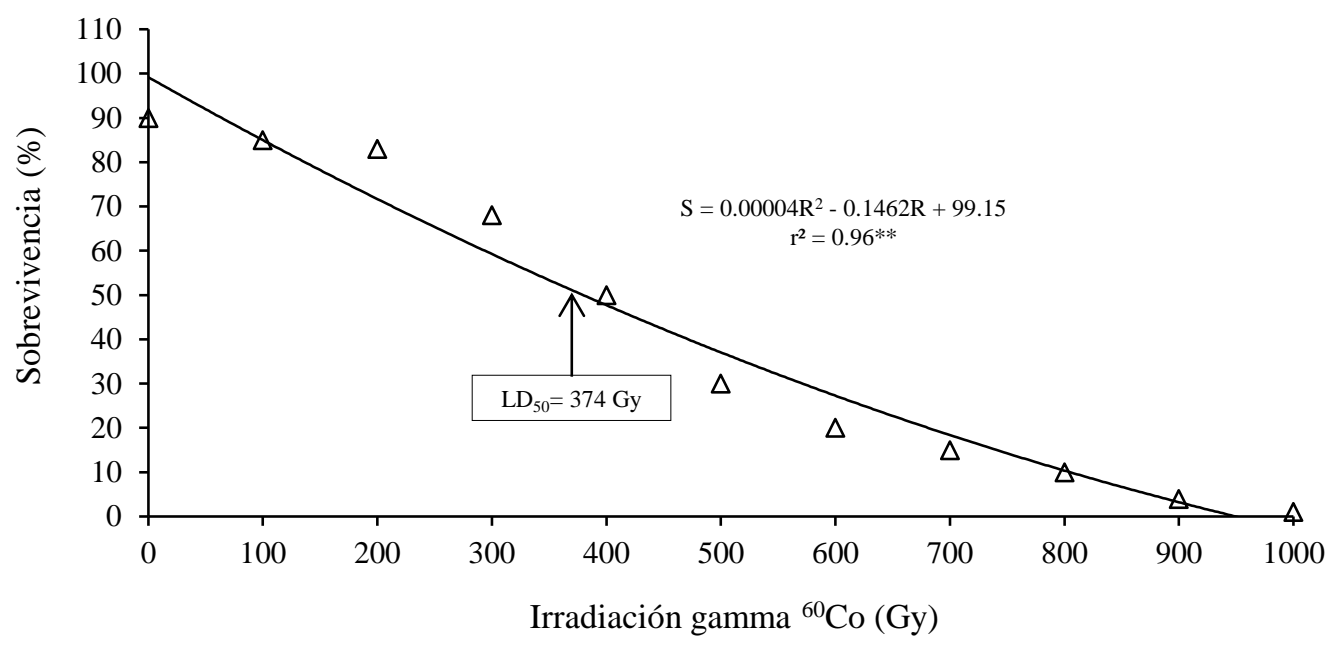

Figura 4. Dinámica de la sobrevivencia y $L^{50}$ en plántulas de girasol cv Periquero (Helianthus annuus L.), provenientes de aquenios irradiados con gamma de ${ }^{60} \mathrm{Co}$, a los $30 \mathrm{dds}$. Instituto Nacional de Investigaciones Nucleares, 2019.

\section{Variabilidad genética en longitud y volumen de raíz}

El análisis de varianza, así como la prueba de comparación múltiple en variabilidad genética, para longitud y volumen de raíz en plántulas de girasol, se presenta en el (Cuadro 3), se puede apreciar que existieron diferencias altamente significativas para ambas variables. Para longitud de raíz, el coeficiente de variación muestra que los datos resultaron ser muy confiables $4.23 \%$, mientras que, para volumen, fueron confiables con $19.96 \%$.

Cuadro 3. Análisis de varianza y prueba de comparación múltiple en girasol (Helianthus annuus L.), $c v$ Periquero, sometido a once niveles de irradiación gamma de ${ }^{60} \mathrm{Co}$. Instituto Nacional de Investigaciones Nucleares, 2019.

\begin{tabular}{ccc}
\hline Irradiación ${ }^{60}$ Co Gy & LR $(\mathrm{cm})$ & VR $\left(\mathrm{cm}^{3}\right)$ \\
\hline 0 & $6.2 \mathrm{~b}$ & $2.1 \mathrm{~b}^{\mathrm{qI}}$ \\
100 & $5 \mathrm{c}$ & $1.6 \mathrm{c}$ \\
200 & $8 \mathrm{a}$ & $2.5 \mathrm{a}$ \\
300 & $3.4 \mathrm{~d}$ & $1.2 \mathrm{~d}$ \\
400 & $2.6 \mathrm{e}$ & $0.8 \mathrm{e}$ \\
500 & $2.3 \mathrm{f}$ & $0.5 \mathrm{f}$ \\
600 & $2.1 \mathrm{~g}$ & $0.5 \mathrm{f}$ \\
\hline
\end{tabular}




\begin{tabular}{ccc}
\hline Irradiación ${ }^{60}$ Co Gy & LR $(\mathrm{cm})$ & VR $\left(\mathrm{cm}^{3}\right)$ \\
\hline 700 & $1.6 \mathrm{~h}$ & $0.3 \mathrm{f}$ \\
800 & $1.4 \mathrm{i}$ & $0.3 \mathrm{f}$ \\
900 & $0 \mathrm{j}$ & $0 \mathrm{~g}$ \\
1000 & $0 \mathrm{j}$ & $0 \mathrm{~g}$ \\
$\mathrm{DSH}$ & $0.187^{* *}$ & $0.26^{* *}$ \\
$\mathrm{CV}(\%)$ & 4.23 & 19.96 \\
\hline
\end{tabular}

$\mathbb{I}=$ medias dentro de columnas con la misma literal, estadísticamente son iguales según Tukey a $p \leq 0.05 ;$ LR=longitud de raíz; $\mathrm{VR}=$ volumen de raíz; $\mathrm{DSH}=$ diferencia significativa honesta; $\mathrm{CV}=$ coeficiente de variación; **; *; ns= significativo al $0.01 ; 0.05$ y no significativo.

La aplicación de 200 Gy para ambos casos resultó de manera positiva, induciendo una mayor longitud y volumen de raíz, con $8 \mathrm{~cm}$ y $2.5 \mathrm{~cm}^{3}$ respectivamente, superando a todos los tratamientos incluido el testigo. Los valores más bajos, ocurrieron con la aplicación de 900 y 1 kGy, debido a que el sistema radical no logró un desarrollo óptimo comparado con los tratamientos donde se aplicó una menor dosis de irradiación. La aplicación de 200 Gy en el cultivo de girasol, puede inducir cambios morfológicos, incrementando la longitud de la raíz axonomorfa y de igual modo, aumenta el volumen del sistema radical, lo que puede traducirse, en un mayor anclaje de la planta al suelo, así como una mayor absorción de agua y nutrimentos.

En un estudio con irradiación gamma en semillas de Leucaena leucocephala Cepero et al. (2001), mencionan que uno de los órganos de la planta más sensible al efecto de la irradiación es la raíz, disminuyendo la longitud hasta en $10 \%$, cuando se aplican 100 Gy. Por su parte Ramírez et al. (2006), al incrementar la dosis de rayos gamma en cuatro variedades de Solanum Lycopersicum L. observaron que 5 y 20 Gy, estimulan la longitud de raíz en esta especie, contrastando con esta investigación.

\section{Análisis de correlación}

El análisis de correlación para las variables morfológicas, altura de planta, longitud y volumen de raíz, se presenta en el Cuadro 4. En él, se puede observar que las variables: longitud y volumen de raíz, altura de planta, se correlacionaron positivamente, resultando además altamente significativas. Por su parte las variables: longitud y volumen de raíz, solo resultaron significativas, de igual modo correlacionándose positivamente. Estos resultados concuerdan con los reportados por Barrios et al. (2014), quienes reportan que el volumen y longitud de raíz, se correlacionan alta y significativamente, a pesar de tratarse de especies diferentes.

Cuadro 4 Análisis de correlación para tres variables morfológicas en girasol (Helianthus annuus L.), $c v$ Periquero. En función de once niveles de irradiación gamma de ${ }^{60} \mathrm{Co}$. Instituto Nacional de Investigaciones Nucleares, 2019.

\begin{tabular}{cccc}
\hline Variables & AP & LR & VR \\
\hline AP & 1 & $0.949^{* *}$ & $0.952^{* *}$ \\
LR & $0.949^{* *}$ & 1 & $0.98^{* *}$ \\
VR & $0.952^{* *}$ & $0.99^{* *}$ & 1 \\
BT & 0.622 & $0.778^{*}$ & $0.756^{*}$ \\
\hline
\end{tabular}

$\mathrm{AP}=$ altura de planta; $\mathrm{LR}=$ longitud de raíz; $\mathrm{VR}=$ volumen de raíz; ${ }^{* *} ;{ }^{*} ; \mathrm{ns}=$ significativo al $0.01 ; 0.05$ y no significativo. 


\section{Dinámica de la biomasa total en plántulas de girasol}

La dinámica de la biomasa seca aérea en plántulas de girasol se presenta en la Figura 4. Se puede apreciar que esta tuvo una tendencia a aumentar de 0 a 45 dds, para todos los niveles de irradiación bajo estudio, asílos máximos valores se alcanzaron en el periodo de 30 a 45 dds. La mayor biomasa, se alcanzó en el testigo $0.7 \mathrm{~g}_{\text {planta }}{ }^{-1}$, seguido de los tratamientos 100 y $200 \mathrm{~Gy}$, quienes acumularon una biomasa seca de 0.45 y $0.4 \mathrm{~g}_{\text {planta }}{ }^{-1}$ respectivamente. Por el contrario, la menor acumulación de biomasa por efecto de la radiación gamma, se presentó en las dosis altas $900 \mathrm{y}$ $1000 \mathrm{~Gy}$, acumulando así solo 0.4 y $0.35 \mathrm{~g} \mathrm{planta}^{-1}$.

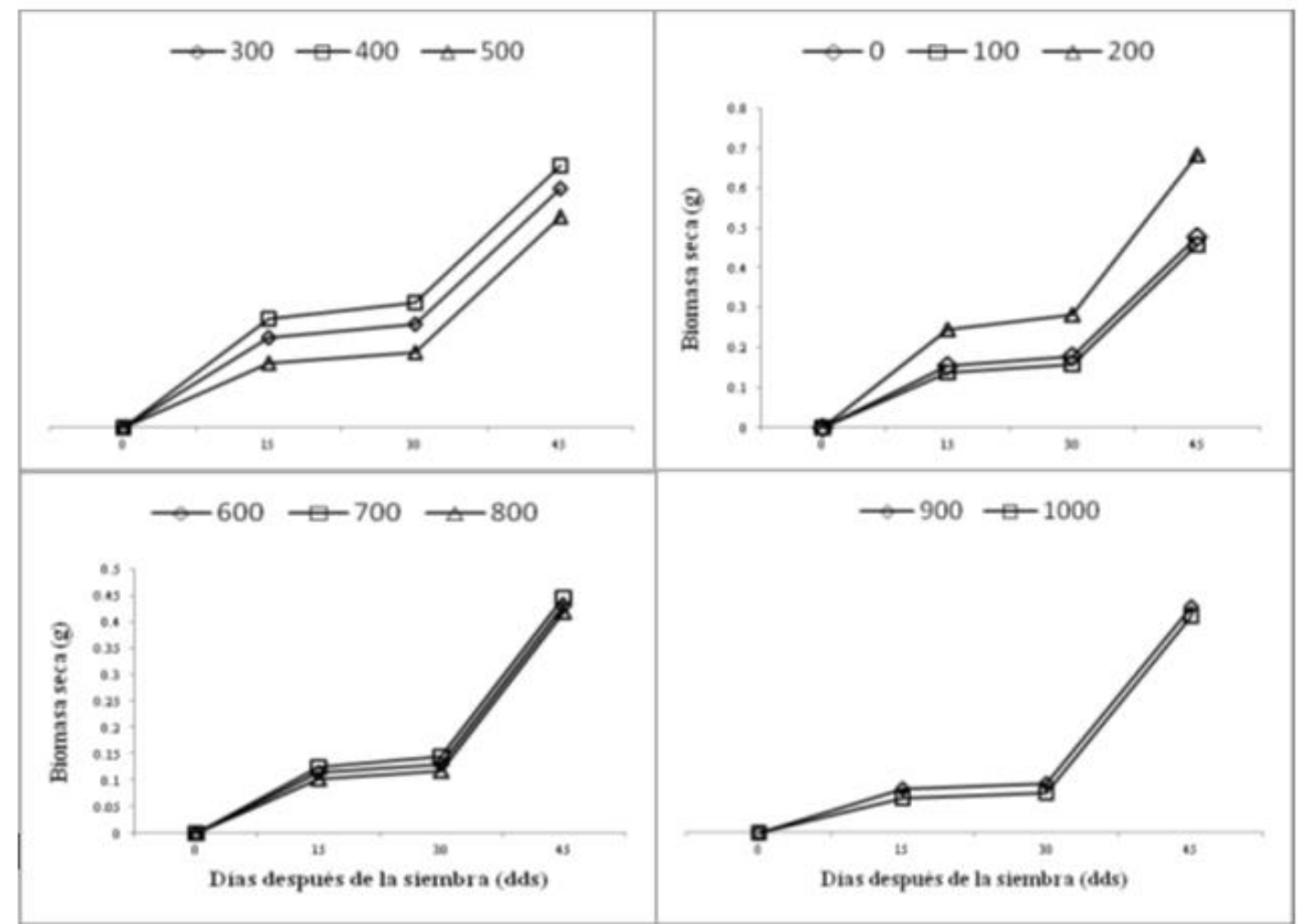

Figura 4. Dinámica de la biomasa seca total en plántulas de girasol (Helianthus annuus L.) cv Periquero. Sometidas a once niveles de irradiación 0, 100, 200, 300, 400, 500, 600, 700, 800, 900 y 1000 Gy con gamma de ${ }^{60} \mathrm{Co}$, a 15, 30 y 45 días después de la siembra. Instituto Nacional de Investigaciones Nucleares, 2019. dds= días después de la siembra.

La menor acumulación de biomasa seca en las plántulas por efecto de la irradiación ha sido estudiada por Yoon et al. (2014), quienes mencionan que las células que conforman los tejidos de la plántula, se dañan al aumentar la dosis de radiación gamma, impidiendo que el mecanismo de reparación de ADN celular, realice su labor, reparando los daños causados. Por su parte Pavan et al. (2013), mencionan que la disminución de biomasa en plántulas sometidas a altas dosis de radiación se debe a la producción de radicales libres en el protoplasto, los cuales ocasionan desordenes metabólicos en la célula, provocando así alteraciones en el patrón de expresión genética, que regulan las rutas metabólicas y sistemas de defensa. 


\section{Unidades SPAD}

Las unidades SPAD presentaron una tendencia a disminuir, a medida que la dosis de irradiación se incrementó, ajustándose los datos a un modelo lineal, resultando ser altamente significativo. Así, el coeficiente de determinación presentó un valor de $96 \%$, indicando que $96 \%$ de las Unidades $\mathrm{SPAD}$, se debieron al incremento en la radiación gamma de ${ }^{60} \mathrm{Co}$ (Figura 5).

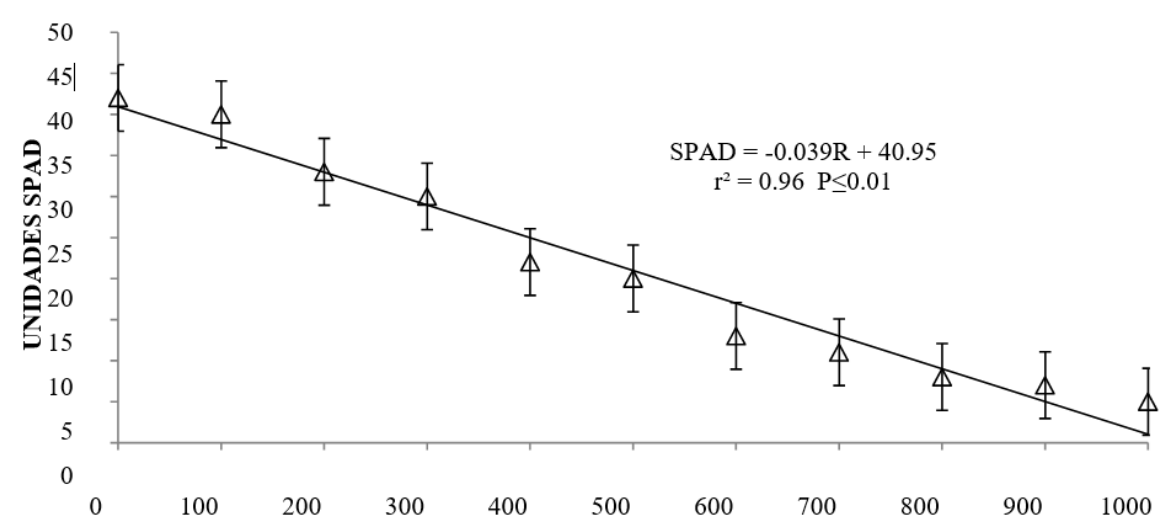

Figura 5. Irradiación gamma de ${ }^{60} \mathrm{Co}$. Unidades SPAD en plántulas de girasol (Helianthus annuus L.) cv Periquero, a 45 dds, sometidos a once niveles de irradiación $0,100,200,300,400,500$, $600,700,800,900$ y 1000 Gy con gamma de ${ }^{60} \mathrm{Co}$. Instituto Nacional de Investigaciones Nucleares, 2019.

La pendiente del modelo, -0.039 indica que, por cada Gy de irradiación aplicado a los aquenios de girasol, las unidades SPAD disminuyen en un 0.039, presentando así una clorosis. Los datos aquí presentados coinciden con los reportados por Antúnez et al. (2017), quienes reportan valores de unidades SPAD de 39.48, para el tratamiento testigo. Fisiológicamente este efecto clorótico, se debió a la detención del ciclo celular en la face G2/M durante la división mitótica de las células somáticas en los nomófilos (Patil et al., 2015).

Este comportamiento también ha sido reportado por Estrada et al. (2011), quienes mencionan que las hojas pálidas (cloróticas), en nardo (Polianthes tuberosa L.), se presentan cuando los bulbos de esta geófita son sometidos a 25 y $30 \mathrm{~Gy}$, de irradiación gamma con ${ }^{60} \mathrm{Co}$.

\section{Conclusiones}

Los modelos matemáticos para germinación, brotación y sobrevivencia, en plántulas de girasol, sometidas a diferentes niveles de irradiación gamma de ${ }^{60} \mathrm{Co}$, se ajustaron a modelos de segundo orden. La máxima $\mathrm{LD}_{50}$, se presentó en orden decreciente para porcentaje de sobrevivencia, germinación y brotación. La radiación gamma de ${ }^{60} \mathrm{Co}$, puede inducir variabilidad genética en plántulas de girasol, en dosis de 100 y 200 Gy, estimulando tanto el volumen y longitud de raíz. La biomasa total seca, disminuyó a medida que aumento la irradiación, siendo dosis de 900 y 1000 Gy, donde la plántula acumuló menor biomasa. La aplicación de irradiación de 700 a 1000 Gy, provocó que las unidades SPAD disminuyeran por efecto de la clorosis. Para estimular variabilidad genética en el sistema radical del girasol $c v$ Periquero, se debe aplicar irradiación gamma de ${ }^{60} \mathrm{Co}$ en dosis de 100 y 200 Gy. 


\section{Agradecimientos}

Los autores agradecen al personal técnico del irradiador Gamma del Instituto Nacional de Investigaciones Nucleares (ININ), por el apoyo para la irradiación del material genético.

\section{Literatura citada}

Álvarez, H. A.; Morales, N. C. R.; Avendaño, A. C. H.; Santellano, E. E.; Melgoza, C. A.; Burrola, B. M. E. y Corrales, L. R. 2018. Dosis letal media y reducción media del crecimiento por radiación gamma en pasto africano (Eragrostis lehmanniana Ness). Ecosistemas y Recursos Agropecuarios. 5(13):81-88. Doi: https://doi.org/10.19136/era.a5n13.1268.

Antúnez, O. O. M.; Cruz, I. S.; Sandoval, V. M.; Santacruz, V. A.; Mendoza, O. L. E.; De la Cruz, T. E. y Peña, L. A. 2017. Variabilidad inducida en caracteres fisiológicos de Physalis peruviana L. mediante rayos gamma ${ }^{60} \mathrm{Co}$ aplicados a la semilla. Rev. Fitotec. Mex. 40(2):211-218. Doi: https://doi.org/10.35196/rfm.2017.2.211-218.

Barrios, M. B.; Buján, A.; Debelis, S. P.; Sokolowski, A. C.; Blasón, A. D.; Rodríguez, H. A.; López, S. C.; De Gracia, J.; Mazo, C. R. y Gagey, M. C. 2014. Relación biomasa de raíz/biomasa total de soja (Glicine max L.) en dos sistemas de labranza. Terra Latinoam. 32(3):221-230.

Cardoso, A.; Crodribb, T.; Lucani, C. DaMatta, F. and McAdam, S. 2018. Coordinated plasticity maintains hydraulic safety in sunflower leaves. Plant, Cell Environ. 41(11): 2567-2576. Doi: https://doi.org/10.1111/pce.13335.

Cepero, L.; Mesa, A. R.; Lajonchere, G. y Prieto, M. 2001. Estimulación del crecimiento de Leucaena leucocephala cv Cunninham con rayos gamma de ${ }^{60} \mathrm{Co}$. Pastos y Forrajes. 24(3):235-240.

Chico, R. J.; Cerna, R. L.; Rodríguez, E. M. y Guerrero, P. M. 2012. Capacidad remediadora de la raíz de girasol, Helianthus annuus L. cuando es sometida a diferentes concentraciones de plomo. REBIOL. 32(2):13-19.

De la Cruz, T. E.; García, A. J. M. and Díaz, L. E. 2019. Slopes comparison of germination and seedling emergence curves VS ${ }^{60} \mathrm{Co}$ gamma radiation dose sunflower achenes. Inter. J. Current Microbiol. Appl. Sci. 8(7):1606-1611. Doi: 10.20546/ijcmas.2019.807.191.

Díaz-López, E.; Báez, I.; Hernández, P. and Loeza-Corte, J. 2017. Potential absorption of calcium in sunflower (Helianthus annuus $\mathrm{L}$.) and its effect on $\mathrm{pH}$ and electrical conductivity. Inter. J. Current Microbiol. Appl. Sci. 6(6):3299-3304. Doi: 10.20546/ijcmas.2017.606.387.

Escalante, E. J. A.; Morales, R. A.; Loeza, C. J. M.; Olivar, H. A.; Rosales, M. B. E.; Vargas, R. E. J. and Díaz, L. E. 2017. Lethal concentration of carbonate of $\mathrm{Ca}$ as a function of the osmotic potential of the solution in sunflower (Heliantus annuus L.). Inter. J. Adv. Eng. Res. Sci. 4(2):103-108. Doi: 10.22161/ijaers.4.2.22.

Estrada, B. J. A.; Pedraza, S. M. E.; De la Cruz, T. E.; Martínez, P. A.; Sáenz, R. C. y Morales, G. J. L. 2011. Efecto de rayos gamma de ${ }^{60} \mathrm{Co}$ en nardo (Polianthes tuberosa L.). Rev. Mex. Cienc. Agríc. 3(3):445-458.

Infante, G. S. y Zárate, G. P. 2012. Métodos estadísticos: un enfoque multidisciplinario. MundiPrensa. $3^{\mathrm{a}} .643 \mathrm{p}$.

Loeza, C. J. M.; Díaz, L. E.; Campos, P. J. M. y Orlando, G. J. I. 2013. Efecto de lignificación de estacas sobre enraizamiento de Bursera morelensis Ram. y Bursera galeottiana Engl. en la Universidad de la Cañada en Teotitlán de Flores Magón, Oaxaca, México. Ciencia Ergo sum. 20(3):222-226. 
Morales, M. E. J.; Morales, R. E. J.; Díaz, L. E.; Cruz, L. A. J.; Medina, A. N. y Guerrero, D. M. 2015. Tasa de asimilación neta y rendimiento de girasol en función de urea y liberación lenta. Agrociencia. 49(2):163-176.

Patil, U. H.; Deshmukh, G. N. and Kazi, N. A. 2015. Mutation breeding in chrysanthemum (Dendranthema grandiflora T.) Asian J. Multidisciplinary Studies. 6(6):230-232.

Pavan, K.; Chaturvedi, A.; Sreedjar, M.; Aparna, M.; Venuvadu, P. and Singhal, R. K. 2013. Gamma radiosensitivity study on rice (Oryza sativa L.). Asian J. Plant Sci. Res. 3(1):54-68.

Ramírez, R.; González, L. M.; Camejo, Y.; Zaldivar, N. y Fernández, Y. 2006. Estudio de radiosensibilidad y selección del rango de dosis estimulantes de rayos $\mathrm{X}$ en cuatro variedades de Tomate (Lycopersicum esculentum Mill.). Cultivos Tropicales. 27(1):63-67.

Steel, D. R. G. y Torrie, J. H. 1996. Bioestadística: principios y procedimientos. Mc Graw Hill. $2^{\mathrm{a}}$ (Ed.). 640 p.

Suaña, Q. M. E. 2018. Capacidad del girasol (Helianthus annuus L.) para absorber cadmio de suelos contaminados en ambiente controlado, Puno. Rev. de Investigaciones de la escuela de Postgrado, Universidad Nacional del Altiplano. 7(1):393-401.

Taiz, L. and Zeiger, E. 2000. Plant physiology. McGrall-Hill. $3^{\mathrm{a}}$ (Ed.). 675 p.

Yoon, A.; Kamaludin, A.; Tajudin, K.; Hazmi, Z.; Bakar, D.; Nezhadahmadi, A. and Golam, F. 2014. The contribution of muslim scientist in botanical science: studies on the using of gamma rays for ginger plants (Zingiber officinale L.). Stem Cell. 5(4):88-94. 